\title{
Transurethral neo-orifice (TUNO) a novel technique for management of upper pole obstruction in infancy
}

\author{
Hubert S. Swana, Tariq S. Hakky, Mark A. Rich \\ Nemours Children's Clinic Orlando (MAR) and Department of Urology, University of South Florida \\ School of Medicine (HSS, TSH), Tampa, Florida, USA
}

\section{ABSTRACT}

Introduction and Objective: Ureteral duplication is the most common urologic abnormality. The upper pole ureter can sometimes be associated with a ureterocele. In rare cases the ureteral insertion is extravesical and can result in significant hydroureteronephrosis. Patients can present with urinary tract infection, abdominal mass or urinary obstruction. Traditional procedures include ureteral reimplantation, ureteroureterostomy or heminephroureterectomy. These reconstructive procedures are technically challenging in small infants, especially when the hydroureteronephrosis is severe. In some cases a distal cutaneous ureterostomy is performed for immediate drainage followed by definitive surgery when the child is older. We describe our initial experience with a novel cystoscopic technique which provides drainage of the upper pole ureter and avoids the need for an incision or stoma.

Materials and Methods: A 3 month-old boy presented with urinary tract infections and failure to thrive. Ultrasound revealed severe upper pole hydroureteronephrosis. Voiding cystourethrography did not reveal vesicoureteral reflux or the presence of a ureterocele. The patient underwent cytoscopy. The ectopic ureteral orifice was not identified. A transurethral, transvesical needle puncture and confirmatory ureteropyelography was used to access the dilated upper pole ureter. Guidewire passage, followed catheter dilation then allowed creation of a new ureteral orifice using a holmium laser.

Results: The patient tolerated the procedure well. He was discharged after overnight observation. The hydronephrosis improved, urinary tract infections have not recurred and the patient rapidly improved feeding and weight gain.

Conclusion: Trans-Urethral Neo-Orifice creation is a minimally invasive option for initial decompression for patients with obstructive ureteral ectopia.

\section{ARTICLE INFO}

Available at: www.brazjurol.com.br/videos/january_february_2013/Swana_143_144video.htm

Int Braz J Urol. 2013; 39 (Video \#2): 143-4

Submitted for publication:

June 16, 2012

Correspondence address:

Dr. Hubert S. Swana

Nemours Children's Clinic Orlando

1717 S. Orange Ave, Suite 100

Accepted after revision:

December 25, 2012

Orlando, Florida, 32806, USA

E-mail: hswana@nemours.org 


\section{EDITORIAL COMMENT}

In this video by Swana and colleagues, the authors highlight a novel endoscopic technique of managing an ectopic ureteral orifice not visualized on cystoscopy. By accessing the dilated upper pole ureter, a neoureteral orifice is thereafter created using a Holmium laser "cutdown" technique. I must firstly congratulate the authors on employing a completely minimally invasive endoscopic approach to a complex condition which would traditionally require an extensive surgical approach. The sequelae of surgery are particularly meaningful when pertaining to a pediatric patient population hence insuring the long-term success of the currently described technique is essential prior to promoting the presently described surgical approach as a definitive and first line therapeutic choice. In conclusion, I would encourage the authors to report intermediate and long-term results (i.e. 1, 2 , and 5 years) of the present surgical approach using functional renal studies (e.g. renal scans) in subsequent years.

Dr. Philippe E. Spiess Associate Professor, Department of Urology Moffitt Cancer Center; Tampa, FL Video Section Editor, International Brazilian Journal of Urology 